\title{
Conocimientos alimentarios de vegetarianos $y$ veganos chilenos
}

\author{
Food knowledge of chilean vegetarians \\ and vegans
}

\begin{abstract}
Background: Vegetarian diets are becoming every day more popular due the increase of health concerns, moral motives and animal respect. Objective: To determinate through an online survey the vegetarian and vegan reasons to follow this type of feeding and their nutritional knowledge about the vegetarian diet. Subjects and methods: 319 surveys were done, 266 subjects declared themselves as vegetarians and 53 subjects as vegans. The main source of nutritional information in those groups was Internet (45\%) and the main reason for being a vegetarian or vegan were moral principles (74\%). In terms of nutritional knowledge and food sources, the vegans were more informed than the vegetarian population. Both groups did not know the consequences of micronutrients deficiencies and food sources of omega 3, vitamin D, vitamin B-12, zinc and iron. Conclusions: Vegetarians and vegans have insufficient knowledge to reach a balanced diet and to avoid micronutrients deficiencies. Health professionals should be prepared in this area to give adequate nutritional advice.
\end{abstract}

Key words: vegetarian, vegan, vitamin B12, iron, lifestyle.

\section{INTRODUCCIÓN}

El ser humano en el transcurso de su evolución ha cambiado la percepción de la comida llevándola desde una mera herramienta de sobrevivencia hacia una instancia en la que se ven fuertemente involucradas ciertas prácticas, ritos, creencias y valores. Esto ha llevado a una conjugación de aspectos sociales que implican el no consumo de carne animal y sus derivados. Entre las razones mencionadas más a menudo para explicar esta elección alimentaria, se refieren el precio de la carne, el desagrado por su sabor y por aspectos de salud (1). El vegetarianismo en Chile y el mundo es una tendencia alimentaria que excluye los alimentos de origen animal o parte de ellos, los veganos en cambio no consumen productos de origen animal (2) y sus derivados. Ambas tendencias han ido en aumento los últimos años demostrándose un auge del comercio enfocado a este público.

Las personas que siguen estas dietas se someten a posibles deficiencias de macro y micronutrientes, debido a ello, buscan apoyo e información de diversas fuentes; profesionales de la salud, datos bibliográficos y medios de comunicación masivos. En nuestro país no existen datos específicos que reflejen los conocimientos adquiridos por la población vegetariana para llevar una dieta equilibrada que satisfaga sus necesidades nutricionales.
Jerusa Brignardello G. Lisu Heredia P. María Paz Ocharán S. Samuel Durán A.
Carrera de Nutrición y Dietética, Facultad de Medicina, Universidad del Desarrollo
Clínica Alemana, Santiago, Chile
Dirigir la correspondencia a:
Profesora
Jerusa Brignardello G.
Carrera de Nutrición y Dietética
Facultad de Medicina
Universidad del Desarrollo-Clínica Alemana
Av. Las Condes 12587 - Lo Barnechea
E-mail: jerusa.brignardello@gmail.com

Este trabajo fue recibido el 29 de Enero de 2013 y aceptado para ser publicado el 5 de Mayo de 2013.
Las personas vegetarianas refieren razones económicas, consideraciones éticas o creencias religiosas como fundamentos para seguir este modelo alimentario. Entre los argumentos más frecuentes para elegir una dieta vegetariana se incluyen la preocupación por el medio ambiente y más específicamente por factores relativos al bienestar animal y consideraciones de salud, en general esta dieta se asocia a mayores beneficios para la salud por la composición de los lípidos y la mayor ingesta de fibra dietética $(3,4)$, la eliminación de la carne roja se ha asociado a una disminución del riesgo de desarrollar enfermedades cardiovasculares (4) y presentan finalmente mejores estilos de vida $(6,7)$.

La evidencia avala este último aspecto ya que comparados con los no vegetarianos, los vegetarianos occidentales tienen un IMC menor, valores de colesterol plasmático total disminuidos y una mortalidad más baja por infarto cardíaco (8). No así en muertes por cáncer y mortalidad total se ha demostrado que una dieta equilibrada que incluya productos de origen animal, remplazando las carnes rojas por blancas y especialmente pescado, agregándole a esto el consumo de cereales no refinados resulta ser tan protectora como una dieta vegetariana (9). En cuanto al riesgo de presentar diabetes tipo 2 , la dieta vegana y vegetariana han mostrado una menor prevalencia en comparación a una alimentación omnívora $(10,11)$. 
Según una encuesta realizada en EEUU, realizada el año 2006 aproximadamente $2,3 \%$ de la población adulta (4,9 millones de personas) seguían habitualmente una dieta vegetariana y afirmaban que nunca comían carne, pescado o aves de corral. En cambio el 1,4\% de la población adulta de Estados Unidos era vegana (12). Otra muestra del creciente interés por el vegetarianismo son el aumento de sitios webs que tratan el tema, la formación de agrupaciones con este interés en común, revistas y libros de cocina, la aparición de restaurantes vegetarianos, comida rápida del mismo tipo, además de un gran aumento de la disponibilidad de productos aptos para vegetarianos; en base a proteína vegetal y alimentos enriquecidos.

En Australia, los estudios demuestran que una proporción significativa de la población está interesada en el vegetarianismo y creen que esta dieta tiene beneficios para la salud asociados, mostrando diversas barreras a las que se ven enfrentados los vegetarianos, entre ellas el consumo de carne por diversión, las preferencias de un vegetariano versus las de su familia y la escasa información disponible. Además de los beneficios asociados a la salud, se determinaron otras causas de adherencia al vegetarianismo, la más recurrente; la defensa de los derechos animales $(13,14)$.

Estudios realizados por la Asociación Dietética Americana y los Dietistas de Canadá concluyeron que una dieta vegetariana correctamente planeada es adecuada en cualquier etapa del ciclo vital. Comparaciones entre vegetarianos y no vegetarianos muestran que los primeros generalmente consumen una mayor cantidad de cereales, semillas, frutos secos, verduras y frutas, y junto a esto la restricción total de productos cárneos, resultando de esta manera una variación de ingesta de nutrientes. Las dietas vegetarianas son usualmente ricas en carbohidratos, ácidos grasos omega 6, fibra dietética, vitamina B12 y zinc, aunque hay estudios que indican menores ingestas de zinc (15), los veganos pueden tener especialmente baja la ingesta de vitamina B12 y calcio $(16,17)$, con la consecuente deficiencia de vitamina B12 en comparación a una dieta omnívora $(18,19)$.

La literatura internacional aporta datos relevantes con respecto a este tema, pero en Chile, existe poca información, sólo recientemente se está llevando a cabo un censo online para recolectar información sobre el numero de vegetarianos existentes en nuestro país (20).

Con el respaldo de diversos estudios que demuestran los beneficios del vegetarianismo, esta problemática cobra importancia a nivel de salud pública, debido a la utilidad que pudieran entregar datos de la situación a nivel nacional para la realización de posibles intervenciones a futuro.

En nuestro país, no se dispone de información sobre los conocimientos de la población vegetariana y vegana con respecto a su dieta, así como el consumo de algunos alimentos y suplementos que buscan satisfacer las necesidades de macro y micronutrientes, debido a la exclusión parcial, para los vegetarianos o total en el caso de los veganos, de alimentos de origen animal cuyos nutrientes pasan a ser críticos a consecuencia de este tipo de dietas, tales como el calcio, hierro, cinc, vitamina B12, vitamina D, riboflavina y ácidos grasos omega-3 de cadena larga.

El objetivo del presente estudio fue determinar mediante una encuestas vía internet las razones de vegetarianos y veganos para seguir este tipo de alimentación y los conocimientos que poseen con respecto a su alimentación.

\section{SUJETOS Y MÉTODOS}

El diseño del presente estudio es de tipo descriptivo, no representativo. La población objetivo fueron hombres y mujeres veganos y vegetarianos, residentes en Chile, entre 16 y 70 años de edad. La recolección de información fue realizada mediante una encuesta on-line en el sitio http:// www.e-encuesta.com. La encuesta se diseñó y redactó con el propósito de recolectar la información necesaria para este estudio, considerando los antecedentes bibliográficos sobre nutrición en vegetarianos y veganos (REF), donde posteriormente la encuesta se validó en estudiantes universitarios.

Cada encuesta fué dirigida específicamente a vegetarianos y a veganos y comprendió un total de 30 preguntas cerradas y abiertas, . La difusión del blog se realizó en redes sociales y en lugares de expendió de alimentos aptos para vegetarianos y veganos. En la página http://encuestavegetarianos.blogspot. com/, en él se detallaron los criterios de inclusión y exclusión para responder la encuesta. Los criterios de inclusión fueron ser mayor de 16 años de edad, de nacionalidad chilena y ser vegetariano o vegano. Se excluyó a aquel sujeto que se declaraba vegetariano, pero consumía carnes blancas (pescado, pollo, pavo).

La encuesta tenía 30 preguntas. Se realizaron 2 encuestas 1 para vegetarianos y 1 para veganos, y buscó indagar en los conocimientos y prácticas de vegetarianos y veganos en nuestro país. Los datos obtenidos fueron analizados en planilla Excel cuantitativamente.

\section{RESULTADOS}

Se obtuvo un total de 319 encuestas, 266 de ellos a vegetarianos y 53 a veganos. El $81 \%$ fueron mujeres y el $19 \%$ hombres de los vegetarianos y $69,8 \%$ y $30,1 \%$, de los veganos. La edad promedio de los encuestados vegetarianos fue 24,3 $\pm 6,7$ años y 23,6 $\pm 5,9$ años para los veganos. En el total de la muestra los consultados presentaron las siguientes características: residir en la Región Metropolitana y ser estudiante (68\%). Dentro de las principales razones que se declararon para seguir el vegetarianismo o veganismo fueron principios morales o "principios animalistas" (74\%), seguido por beneficios para la salud (8,6\%) (tabla 1). La edad promedio de inicio de esta dieta fue 19,4 $\pm 6,7$ años en el caso de los vegetarianos y $19,8 \pm 6,4$ años para los veganos. $76 \%$ de los vegetarianos se declararon ovolactovegetarianos, seguido por lactovegetariano $(10,5 \%)$ y ovovegetariano (9\%), otros $5 \% ; 66 \%$ de los veganos admitió haber seguido una dieta vegetariana antes de optar por el veganismo.

El 75\% de los vegetarianos cree seguir una dieta equilibrada, mientras que $83 \%$ de los veganos sostuvo hacerlo. Al consultar sobre que nutrientes debían cubrir en la dieta por no consumir carne $77,4 \%$ de los vegetarianos tuvo conocimiento, mientras que $92,5 \%$ de los veganos contestó que los nutrientes debían cubrir al no ingerir alimentos de origen animal. En cuanto al reemplazo de las proteinas de fuente animal $87,5 \%$ de vegetarianos y $90,6 \%$ de los veganos indicó sustituirlas, ambos grupos en su mayoría indicaron mediante la ingesta de leguminosas y proteína de soya.

En relación a la principal dificultad para llevar cada tipo de dieta, ambos grupos señalaron que: es el asistir a reuniones sociales para los vegetarianos $34,2 \%$ y para los veganos $23,3 \%$. La segunda dificultad más nombrada por los vegetarianos fue salir a comer a restoranes $23,1 \%$ y $21 \%$ para los veganos fue encontrar alimentos veganos en el mercado. Por otra parte, lograr un aporte de proteínas, hierro, zinc y vitamina B12, fue el aspecto de la dieta que más le preocupaba a todos (70\%), cabe señalar que un bajo porcentaje admitió consumir suplementos alimentarios (26.3\%), siendo menor en los vegetarianos $20,3 \%$ mayor en los sujetos veganos 
65\% En cuanto a la fuente de información sobre estas dietas, vegetarianos y veganos declararon recurrir preferentemente a internet, sólo una minoría refirió dirigirse a nutricionistas y profesionales de la salud, en un porcentaje de 9,4\% y 10,9\% respectivamente (tabla 2 ).

En lo que respecta a los beneficios para la salud de cada dieta, $79 \%$ de los vegetarianos y $88,7 \%$ de los veganos, admitieron conocer aspectos como: disminución del riesgo de padecer cáncer y enfermedades cardiovasculares debido a un aumento del consumo de antioxidantes y una menor ingesta de grasas saturadas, mejora el tránsito intestinal como consecuencia de un mayor aporte de fibra y ayuda a la mantención de un peso saludable.

Referente a las desventajas de seguir cada tipo de dieta, $46 \%$ de los vegetarianos y $42 \%$ de los veganos, seleccionó la necesidad de una adecuada planificación para evitar el déficit de nutrientes, el riesgo de presentar anemia y dificultades a nivel óseo. Por otra parte un porcentaje de ellos aseguró no encontrar desventajas; $6 \%$ y $15 \%$ respectivamente.

En lo que concierne a la importancia del consumo de proteínas, aproximadamente la mitad de vegetarianos $(55,8 \%)$ y el 78,7\% de los veganos, mencionó saber la importancia del consumo diario de proteínas, indicando principalmente funciones de transporte, estructurales, hormonales y de inmunidad.

En cuanto a los omega 3 y micronutrientes (tabla 3), el $89,9 \%$ de vegetarianos y $96,3 \%$ de los veganos, señaló como alimentos de los que puede obtener este nutriente, al aceite de canola y las algas marinas, asegurando en $68,5 \%$ y $75,7 \%$ cada uno, que el riesgo cardiovascular, la dificultad en la visión y los problemas neurológicos al nacimiento son posibles consecuencias de un déficit de éste; $32 \%$ de los veganos y $24 \%$ de los vegetarianos desconocía las consecuencias para la salud de un déficit de ingesta de los omega 3.

Acerca de la vitamina B12, 77,9\% de los vegetarianos y $90,6 \%$ de los veganos, indicó que los alimentos enriquecidos con ella, son una fuente importante de este micronutriente. Además, $71,5 \%$ y $90,6 \%$ respectivamente, reconoció a la anemia y las alteraciones neurológicas como resultado del déficit de ésta; sin embargo, alrededor del 29\% de los vegetarianos consultados refirió simplemente no saber cuáles eran las consecuencias de la carencia de esta vitamina en cambio sólo 9\% de los veganos.

En cuanto a los requerimientos de vitamina D, $77,9 \%$ de los vegetarianos y $94,4 \%$ de los veganos, aseguraron que los alimentos fortificados en ella y la exposición a la luz solar, cubrían sus necesidades, por otra parte, el mayor porcentaje de los encuestados que siguen el vegetarianismo (30,1\%), admitió no saber cuáles son las consecuencias del déficit de vitamina D en la dieta, por el contrario, el grupo restante, afirmó que las patologías a nivel óseo podrían ser producto de una falta de esta vitamina.

Respecto a qué alimentos proveen calcio, 83,1\% de los vegetarianos indicó lácteos, almendras y hortalizas de hojas verdes, y 69,8\% de los veganos respondió que almendras y hortalizas de hojas verdes, aceptando además en 96,3\% y 98,2 correspondiente, que la osteoporosis y las fracturas, son

TABLA 1

Principal motivo para ser vegano o vegetariano.

\begin{tabular}{|c|c|c|c|c|c|c|}
\hline \multirow[t]{2}{*}{ Motivos } & \multicolumn{2}{|c|}{ Vegetarianos } & \multicolumn{2}{|c|}{ Veganos } & \multicolumn{2}{|c|}{ Total } \\
\hline & $\mathrm{N}$ & $\%$ & $\mathrm{~N}$ & $\%$ & $\mathrm{~N}$ & $\%$ \\
\hline Principios animalistas & 197 & 74,1 & 40 & 75,5 & 237 & 74,3 \\
\hline Creencias religiosas & 14 & 5,3 & 0 & 0 & 14 & 4,4 \\
\hline Beneficios para la salud & 23 & 8,7 & 1 & 1,9 & 24 & 7,5 \\
\hline Razones económicas & 1 & 0,4 & 0 & 0 & 1 & 0,3 \\
\hline Desagrado por el sabor de la carne & 10 & 3,8 & 0 & 0 & 10 & 3,1 \\
\hline Otra & 21 & 7,9 & 12 & 22,6 & 33 & 10,3 \\
\hline Total & 266 & 100 & 53 & 100 & 319 & 100 \\
\hline
\end{tabular}

TABLA 2

Principales fuentes de información de vegetarianos y veganos.

\begin{tabular}{|c|c|c|c|c|c|c|}
\hline \multirow[t]{2}{*}{ Fuentes de Información } & \multicolumn{2}{|c|}{ Vegetarianos } & \multicolumn{2}{|c|}{ Veganos } & \multicolumn{2}{|c|}{ Total } \\
\hline & $\mathrm{n}$ & $\%$ & $\mathrm{~N}$ & $\%$ & Total & $\%$ \\
\hline $\begin{array}{l}\text { Nutricionistas u otros } \\
\text { profesionales de la salud }\end{array}$ & 29 & 10,9 & 5 & 9 & 34 & 10,7 \\
\hline Radio, televisión y/o revistas & 3 & 1,1 & 0 & 0 & 3 & 0,9 \\
\hline Internet & 118 & 44,4 & 27 & 51 & 145 & 45,5 \\
\hline Círculo familiar y/o social & 16 & 6,0 & 0 & 0 & 16 & 5,0 \\
\hline Todas las anteriores & 100 & 37,6 & 21 & 40 & 121 & 38 \\
\hline Total & 266 & 100,0 & 53 & 100 & 319 & 100 \\
\hline
\end{tabular}


consecuencias para la salud de la carencia de este mineral.

Sólo $29,3 \%$ de los vegetarianos y $44 \%$ de los veganos, afirmó que se puede obtener zinc de alimentos como frutos secos, cereales integrales y legumbres. Por otro lado, $62,8 \%$ de los vegetarianos y $49,1 \%$ de los veganos desconoce los efectos de la deficiencia de zinc.

Con respecto a la obtención de hierro en la dieta, 46,6\% de vegetarianos y $67,9 \%$ de veganos, refirió que es posible hacerlo a partir de legumbres y hortalizas de hojas verdes. Sin embargo, un porcentaje mayor, $84,6 \%$ de los primeros y $90,6 \%$ de los segundos, declaró saber que la anemia es una patología derivada del déficit de este mineral; $15 \%$ y 9, 4\% de los encuestados desconocía los efectos de la deficiencia de hierro, respectivamente.

\section{DISCUSIÓN}

En Chile y el mundo existe una creciente preocupación por el estado nutricional de la población debido al aumento de las tasas de enfermedades crónicas no transmisibles causadas por el sobrepeso y la obesidad. Las dietas vegetarianas han sido consideradas por expertos como una opción para disminuir los riesgos de presentar estas patologías.

A pesar que se han descrito diversos beneficios de esta dieta, es necesario realizar más estudios sobre el nivel de conocimientos nutricionales que tienen los vegetarianos $y$ veganos y prevenir sus deficiencias.

De acuerdo a los resultados obtenidos, la mayoría del grupo que respondió las encuestas, tanto los vegetarianos como los veganos, fueron mujeres. El grupo etario predominante estaba entre los 16 y 29 años de edad, en ambas encuestas, con un promedio de 24,3 años para vegetarianos y 23,6 para veganos. Este grupo etario tiene un mayor acceso a internet y redes sociales, medios por los cuales se difundió la encuesta; agregado a un creciente interés de las nuevas generaciones por seguir una dieta libre de alimentos de origen animal, siendo los principios animalistas la razón más mencionada resultado que coincide con otro estudio realizado (21). En EEUU, 2\% de los adolescentes entre 6 y 18 años son vegetarianos, de éstos $0,5 \%$ son veganos. En Chile no se conoce esta proporción (22).
Era de esperar en los resultados que un mayor porcentaje de veganos haya seguido una dieta vegetariana anteriormente, correspondiendo esta cifra a $66 \%$.

A pesar de que los encuestados refieren seguir en su mayoría una dieta equilibrada y conocer los beneficios de esta, es importante destacar que en ambos grupos sólo una minoría acudió a los profesionales de la salud y por el contrario, la gran mayoría utilizó internet como fuente principal para obtener la información nutricional, pudiendo ser riesgoso para salud el recurrir a fuentes poco fidedignas.

Un gran porcentaje de los veganos encuestados consumía suplementos alimentarios que contenían vitaminas del complejo $B$, especialmente vitamina B12. Estudios muestran que tanto en omnívoros como en vegetarianos y veganos puede existir deficiencia de vitamina B12, pero suele ser más difícil para estos últimos mantener niveles séricos adecuados (23). La suplementación juega un rol fundamental, sobre todo en la población vegana al tener una dieta más restrictiva, para así poder cubrir los requerimientos de vitamina B12 y lograr una dieta equilibrada que no conlleve riesgo de sufrir anemia y alteraciones neurológicas (24), ya que la dieta vegetariana se ha asociado con un incremento de riesgo de desórdenes mentales (25-27).

En el caso de proteínas una buena alternativa es el amaranto que posee un buen score aminoacídico y buen contenido de proteínas (16\%) (28) al igual que la quinoa (29).

Es conocido que cualquier tipo de dieta, sea omnívora, vegetariana o vegana, si está mal planificada puede producir una deficiencia de macronutrientes y micronutrientes causando problemas para la salud. En el caso de la alimentación que no incluye carne y derivados, se debe tener especial cuidado con la obtención de proteínas con carga aminoacídica completa, vitaminas de complejo B (especialmente vitamina B12) siendo buena fuente productos lácteos, huevo y alimentos fortificados; calcio encontrándose en leche y derivados, frutos secos, cereales integrales, vegetales de hoja verde, crucíferas, legumbres; hierro hallándose en verduras de hoja verde y legumbres. Es importante mencionar que para aumentar la biodisponibilidad del hierro no hemínico de los vegetales la

\section{TABLA 3}

Conocimientos sobre deficiencia y fuentes de nutrientes críticos en veganos y vegetarianos.

\begin{tabular}{|c|c|c|c|c|c|}
\hline & \multicolumn{2}{|c|}{ veganos $(n=53)$} & \multicolumn{2}{|c|}{ vegetarianos $(n=266)$} & \multirow[b]{2}{*}{ valor $\mathrm{p}$} \\
\hline & Sí (\%) & No (\%) & Sí (\%) & No (\%) & \\
\hline Deficiencia de omega 3 & 75,7 & 24,3 & 68,5 & 31,5 & 0,227 \\
\hline Fuentes de omega 3 (correctas) & 96,3 & 3,7 & 89,9 & 10,1 & 0,050 \\
\hline Deficiencia de B12 & 90,6 & 9,4 & 71,5 & 28,5 & 0,001 \\
\hline Fuentes de B12 & 90,6 & 9,4 & 77,9 & 22,1 & 0,004 \\
\hline Deficiencia de vitamina D & 69,9 & 30,1 & 45,2 & 54,8 & 0,001 \\
\hline Fuentes de vitamina D & 94,4 & 5,6 & 77,9 & 22,1 & 0,001 \\
\hline Deficiencia de calcio & 98,2 & 1,8 & 96,3 & 3,7 & 0,396 \\
\hline Fuentes de calcio & 100 & 0 & 95,5 & 4,5 & 0,002 \\
\hline Deficiencia de zinc & 51 & 49 & 37,3 & 62,7 & 0,071 \\
\hline Fuentes de Zinc & 70 & 30 & 63,6 & 36,4 & 0,417 \\
\hline Deficiencia de hierro & 90,6 & 9,4 & 84,6 & 15,4 & 0,182 \\
\hline Fuentes de hierro & 92,5 & 7,5 & 86 & 14 & 0,143 \\
\hline
\end{tabular}

Test chi-cuadrado 
mejor estrategia es consumir alimentos ricos en vitamina $C$ junto con las comidas principales $(29,30)$.

El zinc se encuentra en alimentos como frutos secos, legumbres y los cereales integrales (31); y el omega 3 buena fuente en el aceite de linaza, aceite de canola, nueces, soya, germen de trigo, chia, semilla de colza $(32,33)$. Con respecto a los omegas 3 estudios en vegetarianos muestran menores concentraciones tisulares de ellos (34), que se asocian a un incremento en el riesgo de trastornos depresivos mayores al afectar la funcionalidad neuronal y la plasticidad sináptica $(35,36)$, que a su vez influye en los procesos cerebrales relevantes para la aparición y el mantenimiento de los trastornos mentales (37-39). Además se debe tener en cuenta la importancia de obtener información nutricional de fuentes confiables y considerar el uso de suplementos alimentarios. De acuerdo a lo planteado anteriormente las dietas vegetarianas y veganas no deberían traer consecuencias negativas en el estado nutricional, excluyendo a personas con trastornos absortivos $\mathrm{u}$ otras enfermedades. Por el contrario la evidencia muestra diversos beneficios de estas dietas tales como menor riesgo cardiovascular, disminución de muerte por infartos y menor porcentaje de prevalencia de diabetes tipo $2(25,7,10)$.

\section{CONCLUSIONES}

Existe un elevado porcentaje de veganos y especialmente vegetarianos residentes en Chile que poseen los conocimientos insuficientes para lograr una dieta equilibrada y evitar la carencia de nutrientes, lo que hace necesario que los profesionales de salud estén capacitados en esta área del conocimiento para orientar adecuadamente a estas personas, ya que la principal fuente de información de vegetarianos y veganos es internet.

Se hace hincapié en la necesidad de investigar más sobre el tema con el fin de generar nuevas recomendaciones para la población vegetariana y vegana, en aumento en nuestro país así como en el mundo.

\section{RESUMEN}

Introducción: Las dietas vegetarianas Ilaman la atención cada vez más debido a las crecientes preocupaciones acerca de la salud, principios morales y el respecto hacia los animales. Objetivo: Determinar mediante una encuestas on line las razones de vegetarianos y veganos para seguir este tipo de alimentación y los conocimientos que poseen con respecto a su alimentación. Suejtos y métodos: Se realizaron 319 encuestas a 266 sujetos que se autodeclararon vegetarianos y 53 a veganos. La fuente primaria de información fue internet (45\%), la principal razón para ser vegano o vegetariano es el principio moral (74\%), con respecto a los conocimientos y fuentes alimentarias, los veganos presentan mayores conocimientos que los vegetarianos, aunque ambos presentan importante desconocimiento tanto en las consecuencias de las deficiencias y fuentes alimentarias de omega 3, vitamina $D$, vitamina B12, zinc y hierro. Conclusiones: Los vegetarianos y veganos presentan conocimientos insuficientes para lograr una dieta equilibrada y evitar la carencia de nutrientes, lo que hace necesario que los profesionales de salud estén capacitados en esta área para orientarlos en forma adecuada.

Palabras clave: vegetarianos, veganos, vitamina B12, hierro, estilos de vida.

\section{BIBLIOGRAFÍA}

1. Fiddes $N$. Social Aspects of meet eating. Proceedings of the Nutrition Society 1994; 53 (7): 271-9.

2. Fernandes $K$, Campos F, Flores $H$, Simões $S$, Pereira de
Siqueira L. ALAN. 2010; 60(3): 220-6.

3. Key TJ, Davey G. Prevalence of obesity is low in people who do not eat meat. BMJ. 1996;31:816-7.

4. Farmer B, Larson BT, Fulgoni VL, Rainville AJ, Liepa GU. $A$ vegetarian dietary pattern as a nutrient-dense approach to weight management: an analysis of the national health and nutrition examination survey 1999-2004. J Am Diet Assoc. 2011;111(6):817-27.

5. Lea $E$, Worsley $A$. The factors associated with the belief that vegetarian diet provides health benefits. Asia Pac J Clin Nutr. 2003;12(3):296-303.

6. Craig WJ. Nutrition Concerns and Health Effects of Vegetarian Diets. Nutr Clin Pract. 2010;25:613-20.

7. Key TJ, Appleby PN, Rosell MS. Health effects of vegetarian and vegan diets. Proc Nutr Soc. 2006;65:35-41.

8. Key J, Davey G, Appleby P. Healths benefits of a vegetarian diet. Proc Nutr Soc 1999; (58): 271-5.

9. Dagniele PC. Nutrition and health--potential health benefits and risks of vegetarianism and limited consumption of meat in the Netherlands. Ned Tijdschr Geneeskd 2003; (27):1308-13.

10. Zaman G, Zaman F, Arifullah M. Comparative Risk of Type 2 Diabetes Mellitus Among Vegetarians and Non-Vegetarians. Indian J Community Med. 2010; 3: 441-2.

11. Bolet $M$, Socarrás MM. Alimentación adecuada para mejorar la salud y evitar enfermedades crónicas. Rev Cubana Med Gen Integr. 2010; 26(2): 1561-3038.

12. Craig WJ, Mangels AR. The ADA Position Paper On Vegetarian Diets. J Am Diet Assoc 2009;109(7):1266-82.

13. Regan T. Utilitarianism, Vegetarianism and Animal Rights. Phil Public Affairs 1980; 9(4): 305-24.

14. Lea $E$, Worsley A. Benefits and barriers to the consumption of a vegetarian diet in Australia. Public Health Nutr. 2003; (6): 505-11.

15. Rama Kawade. Zinc status and its association with the health of adolescents: a review of studies in India. Glob Health Action. 2012; 5: 10.3402/gha.v5i0.7353.

16. Haddad H, Berk L, Kettering J, Hubbard R, Peters W. Dietary intake and biochemical, hematologic, and immune status of vegans compared with nonvegetarians. Am J Clin Nutr. 1999; 70(8): 586-98.

17. Key J, Appleby $P$, Rosell M. Health effects of vegetarian and vegan diets. Proc Nutr Soc. 2006; (65): 35-41.

18. Mądry $E$, Lisowska A, Grebowiec P, Walkowiak J. The impact of vegan diet on $B-12$ status in healthy omnivores: five-year prospective study. Acta Sci Pol Technol Aliment. 2012; 2): 209-12.

19. Padró L, Cervera P. Alimentaciones Vegetarianas en la Infancia y la adolescencia. Pediatr Integral 2003;7(5):364-72.

20. Vegetarianos Chile. www.vegetarianoschile.cl Censo Online. Visitada 05 de Octubre de 2012.

21. Bobić J, Cvijetić S, Barić IC, Satalić Z. Personality traits, motivation and bone health in vegetarians. Coll Antropol. 2012;36(3):795-800.

22. Aguirre $M L$, Castillo C, Le Roy C. Desafíos Emergentes en la Nutrición del Adolescente. Rev Chil Pediatr 2010; 81(6): 488-97.

23. Gilsing AMJ, Crowe FL, Lloyd-Wright Z, Sanders TAB, Appleby PN, Allen NE, Key TJ. Blood levels of folate and vitamin $B 12$ in meat-eaters, vegetarians and vegans. Eur $J$ Clin Nutr. 2010; 64 (9):933-9.

24. Forrellat M, Gómis I, Gautier du Défaix H. Vitamina B12: metabolismo y aspectos clínicos de su deficiencia. Rev Cubana Hemato IInmunol Hemoter. 1999;15(3):159-74. 
25. Michalak J, Zhang XC, Jacobi F. Vegetarian diet and mental disorders: results from a representative community survey. Int J Behav Nutr Phys Act. 2012;9:67. doi: 10.1186/14795868-9-67.

26. Herrmann W, Geisel J. Vegetarian lifestyle and monitoring of vitamin B-12 status. Clin Chim Acta. 2002;326:47-59.

27. Kwok T, Cheng G, Woo J, Lai WK, Pang CP. Independent effect of vitamin B12 deficiency on hematological status in older Chinese vegetarian women. Am J Hematol. 2002;70:186-90.

28. Dyner L, Drago S, Piñeiro A, Sánchez $H$, González $R$, Villaamil E . Composición y aporte potencial de hierro, calcio y zinc de panes y fideos elaborados con harinas de trigo y amaranto. Arch Latinoam Nutr. 2007; 57(1): 69-78.

29. WHO/FAO/UNU. Protein and Amino Acid Requirements in Human Nutrition. WHO Techn Report Series 935. Geneva; 2007.

30. Teucher B, Olivares M, Cori H. Enhancers of iron absorption: ascorbic acid and other organic acids. Int J Vitam Nutr Res. 2004;74(6):403-19.

31. Greenberg SM, Tucker RG, Heming AE, Mathues JK. Iron absorption and metabolism. I. Interrelationship of ascorbic acid and vitamin E. J Nutr. 1957; 63(1):19-31.

32. Méndez RO, Bueno K, Campos N, López D, Wyatt CJ, Ortega MI. Contenido total y disponibilidad in vitro de hierro y zinc en alimentos de mayor consumo en Sonora y Oaxaca, México. Arch Latinoam Nutr. 55(2): 187-93.

33. Molina M, África M. Prácticas Dietéticas Vegetarianas. Ámbito Farmacéutico Dietética 2008; 27(10): 80-6.

34. Carrero JJ, Martín-Bautista E, Baró L, Fonollá J, Jiménez J, Boza JJ y López-Huertas E. Efectos cardiovasculares de los ácidos grasos omega-3 y alternativas para incrementar su ingesta. Nutr Hosp. 2005; 20: 63-9.

35. Sanders TA. Meat or wheat for the next millennium? A debate pro veg. Proc Nutr Soc. 1999;58:265-9.

36. Young $G$, Conquer J. Omega-3 fatty acids and neuropsychiatric disoders. Reprod Nutr Dev. 2005;45:1-28.

37. Mandelsamen Percica M, Delas I. Essential fatty acids and psychiatric disorders. Nutr Clin Pract. 2011;26:409-25.

38. Freeman MP. Omega-3 Fatty Acids in Psychiatry: A Review. Ann Clin Psychiatry. 2000;12:159-65.

39. Logan A. Neurobehavioral aspects of omega-3 fatty acids: possible mechanisms and therapeutic value in major depression. Altern Med Ref. 2003;8:410-25. 